\title{
Simulation of 3D Diamond Detectors
}

DOI:

10.1016/j.nima.2016.06.099

\section{Document Version}

Accepted author manuscript

Link to publication record in Manchester Research Explorer

\section{Citation for published version (APA):}

Forcolin, G., Oh, A., \& Murphy, S. (2017). Simulation of 3D Diamond Detectors. Nuclear Instruments \& Methods in Physics Research. Section A: Accelerators, Spectrometers, Detectors, and Associated Equipment , 845, 72-75. https://doi.org/10.1016/j.nima.2016.06.099

\section{Published in:}

Nuclear Instruments \& Methods in Physics Research. Section A: Accelerators, Spectrometers, Detectors, and Associated Equipment

\section{Citing this paper}

Please note that where the full-text provided on Manchester Research Explorer is the Author Accepted Manuscript or Proof version this may differ from the final Published version. If citing, it is advised that you check and use the publisher's definitive version.

\section{General rights}

Copyright and moral rights for the publications made accessible in the Research Explorer are retained by the authors and/or other copyright owners and it is a condition of accessing publications that users recognise and abide by the legal requirements associated with these rights.

\section{Takedown policy}

If you believe that this document breaches copyright please refer to the University of Manchester's Takedown Procedures [http://man.ac.uk/04Y6Bo] or contact uml.scholarlycommunications@manchester.ac.uk providing relevant details, so we can investigate your claim.

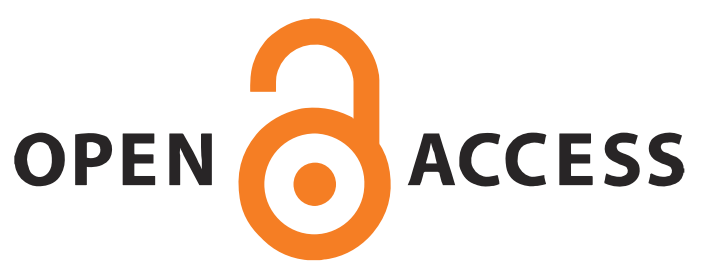




\title{
Simulation of 3D Diamond Detectors
}

\author{
G. T. Forcolin ${ }^{\mathrm{a}, *}$, A. $\mathrm{Oh}^{\mathrm{a}}$, S. A. Murphy ${ }^{\mathrm{a}}$ \\ ${ }^{a}$ School of Physics and Astronomy, University of Manchester, UK
}

\begin{abstract}
3D Diamond detectors present an interesting prospect for future Particle Physics experiments. They have been studied in detail at beam tests with $120 \mathrm{GeV}$ protons and $4 \mathrm{MeV}$ protons. To understand the observations that have been made, simulations have been carried out using Synopsys TCAD in order to explain the movement of charge carriers within the sample, as well as the effects of charge sharing. Reasonable agreement has been observed between simulation and experiment. Keywords: Diamond, 3D Detector, Simulation, TCAD
\end{abstract}

\section{Introduction}

As the LHC enters into the high luminosity domain, increasingly high requirements will be put on the radiation hardness of the detectors used, particularly the semiconductor tracking detectors closest to the interaction points. There are two approaches that can increase the radiation hardness 5 of these detectors:

1. Use of a more radiation tolerant geometry, such as that employed in 3D detectors, increasing radiation hardness as the inter-electrode distance is reduced, meaning that charge carriers are less likely to get trapped. 3D Silicon detectors have already been successfully produced and make up $25 \%$ of the IBL 1 (the new innermost layer of the ATLAS detector at the LHC).

2. Use of chemical vapor deposition (CVD) diamond, which has already been used for radiation and beam monitoring in BaBar, Belle, CDF and the LHC experiments 2. Diamond has a high bond strength (43 eV needed to displace an atom, $13.6 \mathrm{eV}$ for Silicon). Diamond also has a large band gap (5.5 eV compared to $1.12 \mathrm{eV}$ for Silicon) resulting in a low leakage current even after higher irradiations.

15 The aim of 3D diamond detectors is to combine these two approaches by producing diamond detectors with graphitic microwires acting as electrodes drilled into the diamond bulk [3] [4, thus producing detectors that are more radiation resistant than either of the two approaches can achieve individually,

\footnotetext{
${ }^{*}$ Corresponding author
} 
meaning they would be better able to carry on functioning in high radiation environments, such as future LHC upgrades.

3D diamond detectors also present interesting prospects for applications in other fields such as medical physics. A 3D geometry allows the detector to be operated at a low voltage for an equivalent performance, while diamond has the advantage of being a non-toxic tissue equivalent material.

\section{Device Simulation}

Simulations were performed using Synopsys TCAD [5], a semiconductor device simulation package, to understand the behavior of single crystal (scCVD) and polycrystalline (pCVD) 3D Diamond detectors.

In order to simulate a device, it is first of all necessary to create a mesh, modeling the device as a discrete set of nodes. Boundary conditions, such as the electrode voltage, are then applied to the device, and a quasistationary simulation is used to find the steady state behavior.

30 Once the steady state behavior of the device has been determined, some charge is injected into the device to simulate the charge deposited by a particle hit. A time dependent (transient) simulation is then performed on the device to compute the signal observed at the electrodes due to a charged particle hit.

The behavior of the device is modeled by iteratively solving the governing equations of semiconductors (the continuity equations for electrons and holes, as well as the Poisson equation). A field dependent mobility model was also included [6], the parameters used for this model were those measured by Pernegger et al [7].

Simulations were performed in order to better understand the observed behaviour of single crystal (scCVD) 3D diamond detectors at test beams using $120 \mathrm{GeV}[\underline{8}$ and $4 \mathrm{MeV}[9$ protons, while

40 more simulations are required to understand the behavior polycrystalline (pCVD) devices during measurements using $120 \mathrm{GeV}$ protons[10].

\section{3D Single Crystal Minimum Ionizing Particle (MIP) simulations}

To better understand observations made using a scCVD 3D Diamond detector at a test beam with $120 \mathrm{GeV}$ protons [8], a 2x2 array of cells was simulated, with a $150 \mu \mathrm{m}$ pitch and a $500 \mu \mathrm{m}$ detector thickness to match the characteristics of the detector used during the experiment.

Experimental data shows that the charge collection was mostly uniform throughout the detector, with some cells having a greatly reduced signal due to a missing or broken readout electrode.

However it was also observed that in some regions located around bias columns, signals with a negative polarity were observed in the cells adjacent to those containing the hit. 
Simulations were performed to understand this phenomenon; the observations were recreated when a finite charge lifetime of 70ns was introduce to simulate the effect of charge trapping. Regions where negative charges were observed in cells adjacent to the particle hit formed in the simulation around the position of missing or broken bias columns. The signal as a function of position due to a hit in the adjacent cell is shown in (Figure 1) The region where the highest signal is observed is due to charge sharing between cells as this is the region of the cell closest to the electrode where the signal is measured, meaning that for hits in this region, some of the charge has been generated in the neighboring cell. This result shows good agreement with the experimental data [8]. These observations were explained by considering the weighting field in a 3D detector (Figure 22). Ramo's theorem[11] states that the instantaneous current induced at an electrode depends on the component of the weighting field in the direction in which the charge is traveling. As a result of this, in a 3D configuration, it is clear that for a hit in the neighboring cell, charge carriers would have to cross regions where the component of the weighting field in their direction of motion points in opposite directions. Hits at certain positions will therefore result in charge carriers generating bi-polar signals for hits in these regions. These signals normally result in no overall charge collection, however a significant amount of trapping will result in some left over signal, and this can be either positive or negative depending on the position of the hit relative to the electrode.

Normally charge collection in 3D scCVD diamond detectors is very fast, and a large enough number of charge carriers reach the electrodes before they are trapped, meaning that any residual signals would be small and hence difficult to detect. However in this experiment, a missing bias column results in an extended low field region as shown in Figure 3 . This greatly increases the time taken for charge collection to occur, thus allowing more trapping to occur, and the residual signals to become significant.

\section{3D Single Crystal TRIBIC simulations}

Time Resolved Ion Beam Induced Current (TRIBIC) measurements are a novel technique that 75 can be used to produce spatially resolved Transient Current (TCT) measurements[12, in order to map the electric field and the mobility of a detector. Measurements using this technique were performed on a 3D scCVD sample (shown in Figure 4) using $4 \mathrm{MeV}$ protons 9 .

The sample used in the experiment consisted of square cells with a size of $120 \mu \mathrm{m}$ and had a detector thickness of $500 \mu \mathrm{m}$, whereas a $4 \mathrm{MeV}$ proton stops after $\approx 80 \mu \mathrm{m}[13$ in diamond, producing a Bragg peak. The track was therefore modeled in the simulations using a combination of two tracks with different amounts of energy deposited per unit length; a track extending from the surface of the sample to a depth of $80 \mu m$ with a small amount of charge deposited per unit length, 


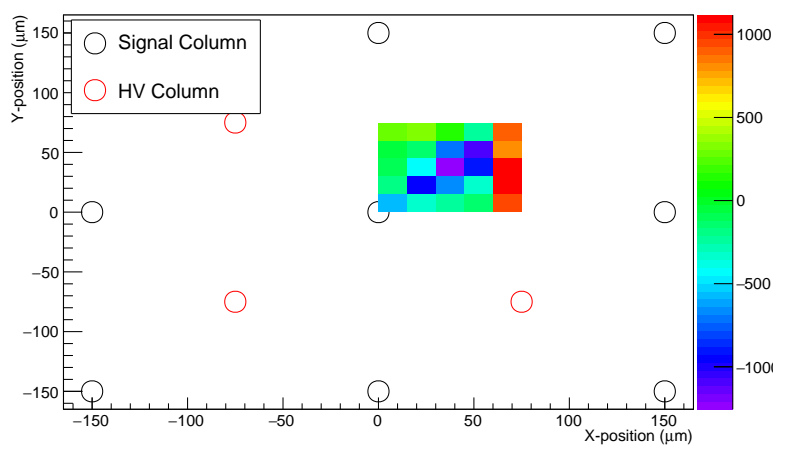

Figure 1: Plot showing the simulated charge collected by the right-most line of signal electrodes due to hits in the the quarter cell between $(0,0)$ and $(75,75)$ when a bias columns is missing from $(75,75)$. The columns are connected together along the $\mathrm{Y}$ direction to match the geometry of the detector used in the experiment.

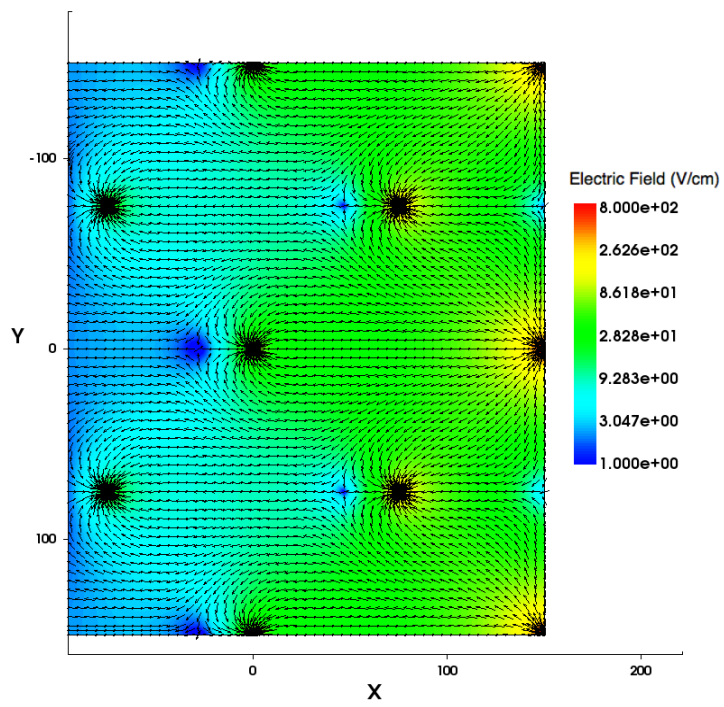

Figure 2: Image showing the magnitude and direction of the weighting field due to a set of connected signal columns, which determines the measured signal according to Ramo's theorem[11]. 


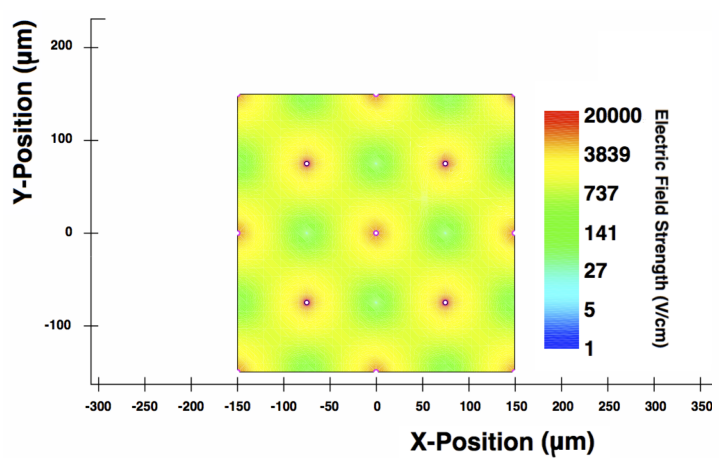

(a)

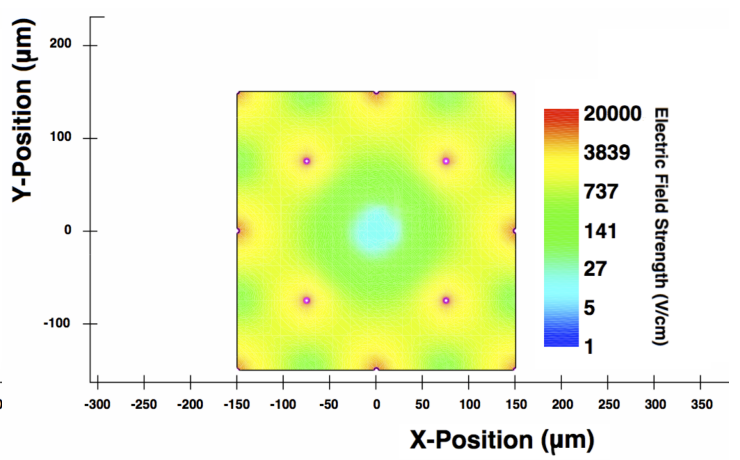

(b)

Figure 3: Plots showing the electric field in a 3D detector with intact electrodes (a) and with a missing or broken electrode (b).

and a short track centered around $80 \mu m$ in depth with a high charge density; the combination of the two summing to the total charge deposited by a $4 \mathrm{MeV}$ proton, with the bulk of the charge deposited at a depth of $80 \mu \mathrm{m}$. The mesh used also had a sample thickness of $500 \mu \mathrm{m}$ and the area simulated comprised a quarter cell, which meant that any effect due to charge sharing and neighboring electrodes was not included.

The amplitude of the current pulse generated by each hit in the experiment was compared to simulated values as a function of position. Figure 5 shows the comparison of two typical curves whereas Figure 6 shows the comparison of the current amplitudes of simulations and experiment (Current amplitudes were used as an analogue for the collected charge as the data was noisy, as can be seen in Figure 5). Both show that there is a strong dependence of the signal amplitude on the position of the hit, and hence on the electric field at that point. The simulations were performed on a quarter cell, and hence do not take into account the effects of neighboring cells, which could account for why the simulations are much more symmetrical than the experimental data.

\section{Future Prospects}

A polycrystalline 3D Diamond detector was also studied at a testbeam using $120 \mathrm{GeV}$ protons and the data is currently being analyzed[10]. Simulations are being performed to understand the charge trapping effects in 3D pCVD detectors, with the aim of understanding how trapping at grain boundaries and in the bulk of the material affects the charge collected.

Work is also currently under way to use simulations to understand how the performance of 3D diamond detectors can be affected by various parameters. The effects of varying the diameter of the 


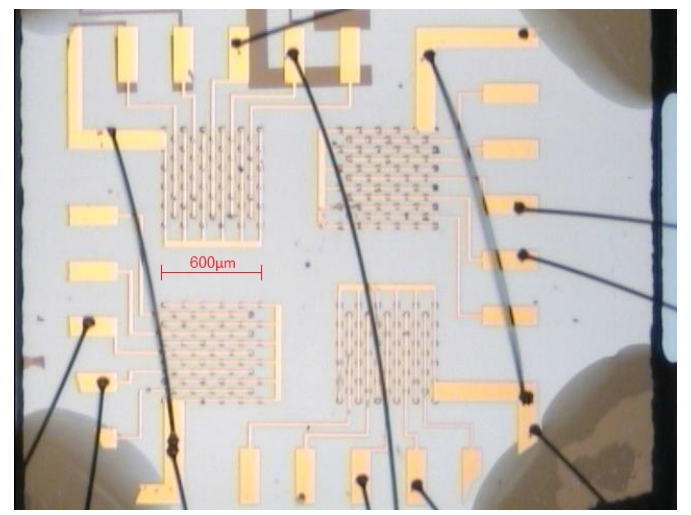

Figure 4: Image showing the detector used for the TRIBIC experiment, which was also simulated.

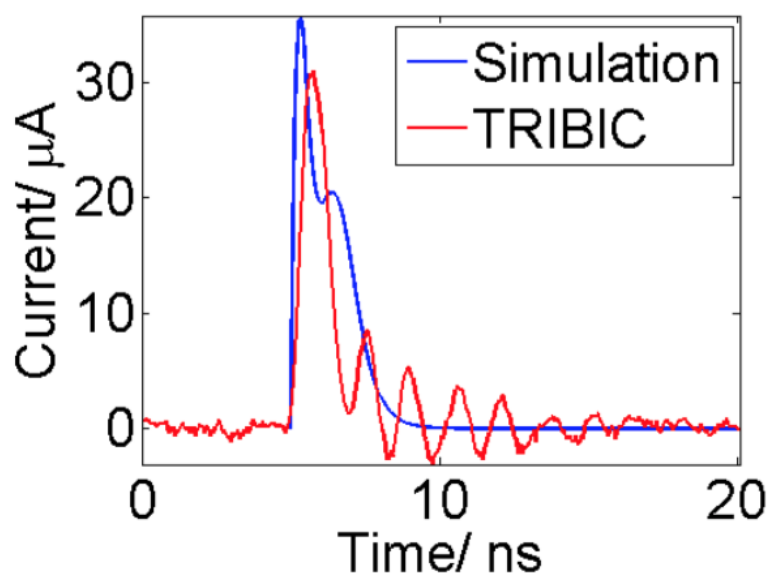

Figure 5: Plot showing a typical comparison of a current pulse generated in the experiment, and a simulated current pulse. The structure visible in the simulated pulse is due to the different arrival times of electrons and holes at the respective electrodes. 


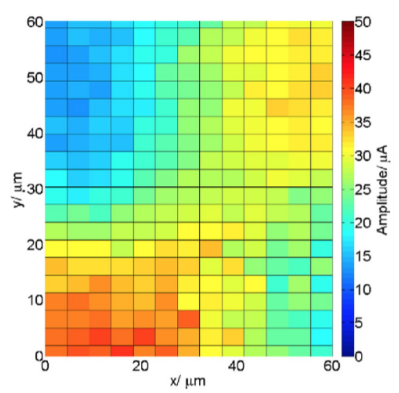

(a)

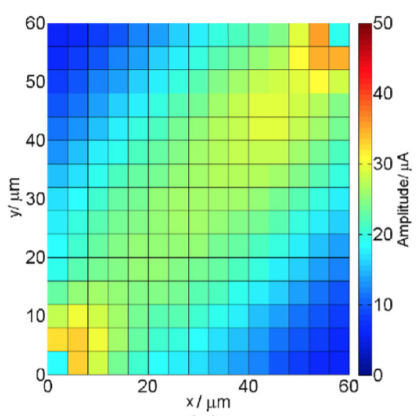

(c)

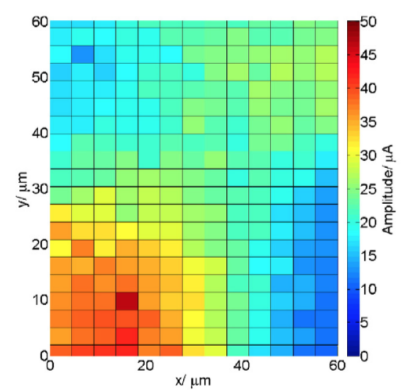

(b)

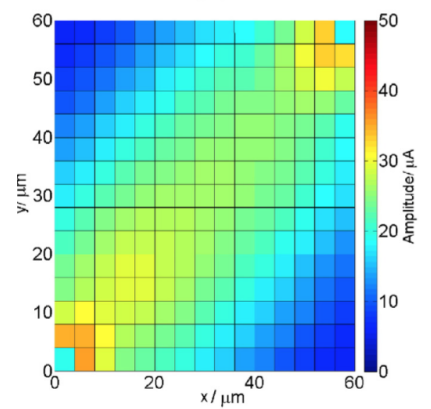

(d)

Figure 6: Plots showing the amplitude of the current pulse due to a $4 \mathrm{MeV}$ proton hit obtained experimentally as a function of position at $+60 \mathrm{~V}$ (a) and $-60 \mathrm{~V}$ (b), as well as the simulated the amplitude of the current pulse obtained by simulation as a function of position at $+60 \mathrm{~V}$ (c) and $-60 \mathrm{~V}(\mathrm{~d})$. The plots represent an area of a quarter cell, where a readout column is located at $(0,0)$ and a bias column is located at $(60,60)$ 
electrodes and the pitch of the device are under investigation, with the aim of finding an optimal combination for the desired application.

Simulations are also being performed to optimize the performance of 3D diamond detectors, by studying different geometries (for example hexagonal cells) to understand how to minimize low field regions between electrodes, as well as understanding how to improve charge collection and maximize the sensitive area of 3D Diamond detectors.

\section{Conclusion}

TCAD has been successfully used to simulate and better understand the behavior of various scCVD 3D diamond devices. Observations of negative signals in single crystal 3D diamond detectors were understood as the result on missing or broken bias columns, creating a large low field region which greatly increased the lifetime of charge collection and hence greatly increased the effect of charge trapping. Simulations also were in agreement with TRIBIC data, showing a strong dependence of the amplitude of the currently pulse generated by a hit on the position of the hit. Work is currently ongoing to better understand the behavior of both pCVD and scCVD devices.

\section{Acknowledgements}

GF and SM would like the acknowledge the support from the STFC.

AO would like to acknowledge support from the Royal Society through grant UF120106.

\section{References}

[1] A. Miucci, The ATLAS insertable b-layer project, Journal of Instrumentation 9 (02) C02018.

[2] D. Dobos, H. Pernegger, Diamond pixel modules and the atlas beam conditions monitor, Nuclear Instruments and Methods in Physics Research Section A: Accelerators, Spectrometers, Detectors and Associated Equipment 628 (1) (2011) 246 - 250, vci 2010 Proceedings of the 12th International Vienna Conference on Instrumentation.

[3] T. Kononenko, M. Komlenok, V. Pashinin, S. Pimenov, V. Konov, M. Neff, V. Romano, W. Lthy, Femtosecond laser microstructuring in the bulk of diamond, Diamond and Related Materials 18 (23) (2009) 196 - 199, jce:title¿NDNC 2008 Proceedings of the International Conference on New Diamond and Nano Carbons 2008i/ce:titlej. doi:http://dx.doi.org/10. 1016/j.diamond.2008.07.014. URL http://www .sciencedirect.com/science/article/pii/S0925963508003981 
q [4] A. Oh, B. Caylar, M. Pomorski, T. Wengler, A novel detector with graphitic electrodes in

घ $\{$ CVD $\}$ diamond, Diamond and Related Materials 38 (0) (2013) 9 - 13. doi:http://dx.doi. org $/ 10.1016 / j . d i a m o n d .2013 .06 .003$. URL http://www.sciencedirect .com/science/article/pii/S0925963513001106

[5] http://www.synopsys.com/home.aspx (15 March 2016).

[6] D. M. Caughey, R. E. Thomas, Carrier mobilities in silicon empirically related to doping and field, Proceedings of the IEEE 55 (12) (1967) 2192-2193.

[7] H. Pernegger, S. Roe, P. Weilhammer, V. Eremin, H. Frais-Klbl, E. Griesmayer, H. Kagan, S. Schnetzer, R. Stone, W. Trischuk, D. Twitchen, A. Whitehead, Charge-carrier properties in synthetic single-crystal diamond measured with the transient-current technique, Journal of Applied Physics 97 (7).

[8] F. Bachmair, L. Baeni, P. Bergonzo, B. Caylar, G. Forcolin, I. Haughton, D. Hits, H. Kagan, R. Kass, L. Li, A. Oh, S. Phan, M. Pomorski, D. Smith, V. Tyzhnevyi, R. Wallny, D. Whitehead, A 3d diamond detector for particle tracking, Nuclear Instruments and Methods in Physics Research Section A: Accelerators, Spectrometers, Detectors and Associated Equipment 786 (2015) $97-104$.

[9] G. Forcolin, V. Grilj, B. Hamilton, L. Li, M. McGowan, S. Murphy, A. Oh, N. Skukan, D. Whitehead, A. Zadoroshnyj, Study of a 3d diamond detector with photon and proton micro-beams, Diamond and Related Materials 65 (2016) 75 - 82, 26th International Conference on Diamond and Carbon Materials.

[10] F. Bachmair, et al., Test beam results of 3 d detectors constructed with single-crystal and polycrystalline diamond, 14th Vienna Conference on instrumentation - VCI 2016.

[11] S. Ramo, Currents induced by electron motion, Proceedings of the IRE 27 (9) (1939) 584-585.

[12] H. Schone, D. S. Walsh, F. W. Sexton, B. L. Doyle, P. E. Dodd, J. F. Aurand, R. S. Flores, N. Wing, Time-resolved ion beam induced charge collection (tribicc) in micro-electronics, IEEE Transactions on Nuclear Science 45 (6) (1998) 2544-2549. doi:10.1109/23.736496.

[13] http://www.nist.gov (15 March 2016). 